\title{
Optimization strategy of automobile dispatching based on neural network model
}

\author{
Chuyuan Wang ${ }^{1, *}$ \\ ${ }^{1}$ Shanghai University of Electric Power, College of Electrical Engineering, 200090 Shanghai, China
}

\begin{abstract}
As a representative product of the sharing economy era and a powerful supplement to public transportation shared cars have the characteristics of convenience, efficiency, environmental protection, and green travel, and to a certain extent alleviate the contradiction between supply and demand, and solve the problem of long-term idle vehicles and overloaded operation of roads problems. But the uneven distribution of shared cars, the coexistence of no cars, and empty seats will happen. To solve the above problems, this article first analyzes data outliers, data missing values, and data standardization processing on the attached data, and then builds a BP neural network demand prediction model to obtain the distribution of shared car usage in the city.
\end{abstract}

\section{Introduction}

In recent years, the sharing economy has gradually merged with all walks of life [1], resulting in various sharing industries that rely on network platforms. In the management process of car-sharing companies, vehicle parking outlets are a key factor affecting vehicle utilization [2].

As an emerging mathematical modeling method, the neural network has the characteristics of recognizing complex nonlinear systems and is more suitable for realtime scheduling. The paper describes the structure and algorithm of the neural network model-BP neural network [3] and proposes on this basis a real-time scheduling prediction model based on BPNN $[4,5]$.

The large-scale application of electric vehicles has caused a serious impact on the power grid and transportation system. At present, there is a lack of research on electric vehicle charging scheduling strategies based on the performance of the power grid and transportation system. This paper establishes a mathematical model to analyze the distribution of shared car use in the city to formulate the most beneficial shared car scheduling scheme for enterprises.

\section{Data organization}

\subsection{Data preprocess and analysis}

Data preprocessing should mainly review the original data: completeness and accuracy. The completeness audit is mainly to check whether the unit or individual that should be investigated has omissions and whether all the survey items or indicators are filled in completely. Accuracy review mainly includes two aspects: one is to check whether the data truly reflects the objective reality and whether the content is in line with reality; the other is to check whether the data is wrong and whether the calculation is correct, etc.

The data includes five column indicators, each column indicator has its unique meaning, as shown below:

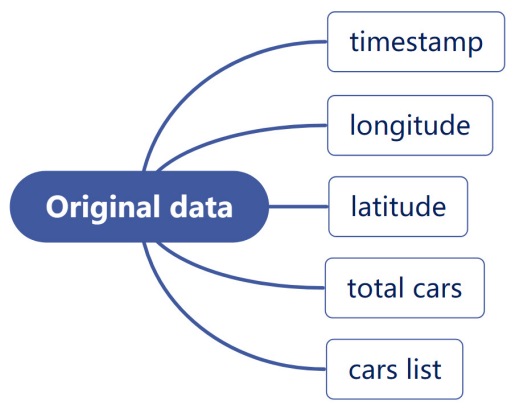

Figure 1. Attachment data analysis.

For the first column indicator, we can know from this indicator that the information provided to us is the time node of this sample data; the second and third column indicators give us latitude and longitude information. These two indicators can determine a specific The location of the shared car parking spot; the fourth column indicator can tell us the number of cars parked at the parking spot; the fifth column indicator can tell us the number of the car parked at the parking spot.

\subsection{Data processing}

We count the null values in each column of the data, and we find that there are no null values in the five columns, so there are no missing values in the attached data. We use excel's filtering function to count the indicator data

\footnotetext{
* Corresponding author: 1253268159@qq.com
} 
ranges of the five columns of indicators in the attached data, as shown in the table 1:

Table 1. Outlier analysis.

\begin{tabular}{|c|c|c|c|c|c|}
\hline Index & timestamp & longitude & latitude & total_cars & carsList \\
\hline Range & $(2018,2019)$ & $(34,35)$ & $(31,33)$ & {$[0,9]$} & {$[0,9]$} \\
\hline
\end{tabular}

From the statistical table data, it is not difficult to find that the data ranges of the five columns of indicators are within the normal range, so there is no abnormal value.

\subsection{Data standardization}

Standardization refers to the unification of repetitive things and concepts in social practices such as economy, technology, science, and management through the formulation, publication, and implementation of standards to obtain the best order and social benefits. In this paper, to constrain the data between $0-1$, we adopt the maximum-minimum normalization process.

$$
z_{i j}=\left|\frac{x_{i j}-\min \left(x_{i j}\right)}{\max \left(x_{i j}\right)-\min \left(x_{i j}\right)}\right|
$$

Among them, $\boldsymbol{z}_{\mathrm{ij}}$ represents the standardized value and represents the value corresponding to the $j$ th sample of the $i$ th index of the original value. The processed variables are pure quantities without units, and their sizes are between 0 and 1 , so the influence of dimensions is eliminated.

\section{BP neural network prediction model construction}

\subsection{BP neural network prediction model}

BP neural network has arbitrarily complex pattern classification capabilities and excellent multidimensional function mapping capabilities, which solves the XOR and some other problems that simple perceptrons cannot solve. Structurally, the BP network has an input layer, a hidden layer and an output layer; in essence, the BP algorithm uses the square of the network error as the objective function and uses the gradient descent method to calculate the minimum value of the objective function. The following is the schematic diagram of the BP neural network.

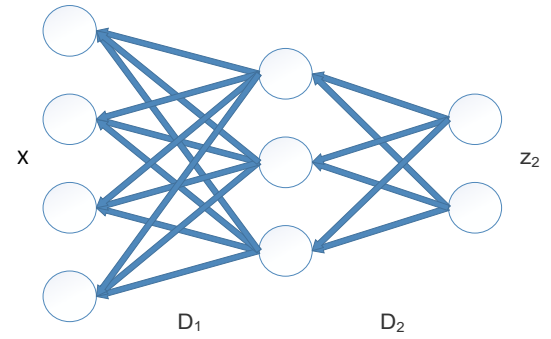

Figure 2. BP neural network training steps.

\subsection{Model construction}

The BP neural network model is a basic system model to solve the problem by analyzing the "weight" of the influence of input information on the output result. Aiming at the highly non-linear variable data in Annex 1, this question uses a supervised learning neural network to predict the stock price loss. The construction process is as follows [2]:

(1) Initialize the BP neural network prediction model: the connection weight $w_{i j}, v_{j t}$, and threshold $\theta_{j}, \gamma_{1}$, of the neural network model are respectively assigned values in the interval $[0,1]$.

(2) Calculate the input $u_{j}$ and $h_{j}$ of the neural nodes of the hidden layer in the BP neural network, namely:

$$
\begin{gathered}
u_{j}=\sum_{i=1}^{n} w_{i j} x_{i}-\theta_{j} \\
h_{j}=f\left(u_{j}\right)=\frac{1}{1+\exp \left(-u_{j}\right)}
\end{gathered}
$$

(3) Calculate the input $\boldsymbol{l}_{l}$ and output $\boldsymbol{y}_{\mathrm{t}}$ of the neural nodes in the output layer of the BP neural network, namely:

$$
\begin{array}{r}
l_{l}=\sum v_{j t} h_{j}-\gamma_{l} \\
y_{t}=\frac{1}{1+\exp \left(-l_{l}\right)}
\end{array}
$$

(4) Calculate the weight error $\varepsilon_{i}$ of the BP neural network connected to the neural node $\mathrm{t}$ of the output layer, namely:

$$
\varepsilon_{i}=\left(c_{t}-y_{t}\right) y_{t}\left(1-y_{t}\right)
$$

(5) Calculate the weight error $\varepsilon_{j}$ of the BP neural network connected to the hidden layer neural node $\mathrm{j}$, namely:

$$
\varepsilon_{j}=\sum_{t=1}^{q} \varepsilon_{i} v_{j t} h_{j}\left(1-h_{j}\right)
$$

(6) Update the connection weight $v_{j t}$ and threshold $\gamma_{t}$ of the BP neural network,

$$
\begin{gathered}
w_{j t}(N+1)=w_{j t}(N)+\partial \varepsilon_{j} x_{i} \\
\theta_{j}(N+1)=\theta_{j}(N)+\beta \varepsilon_{j}
\end{gathered}
$$

(7) Calculate the error between the output value and the expected value. If the set accuracy requirement is met, the neural network training and learning ends, otherwise, go to step (2) to continue training and learning. 


\section{BP neural network demand prediction solution}

We randomly selected 1000 different latitude and longitude points from the attached data and predicted the number of car parking demands at the parking point at 2019-01-08 02:27:28. The BP neural network prediction result is shown in Figure 3.
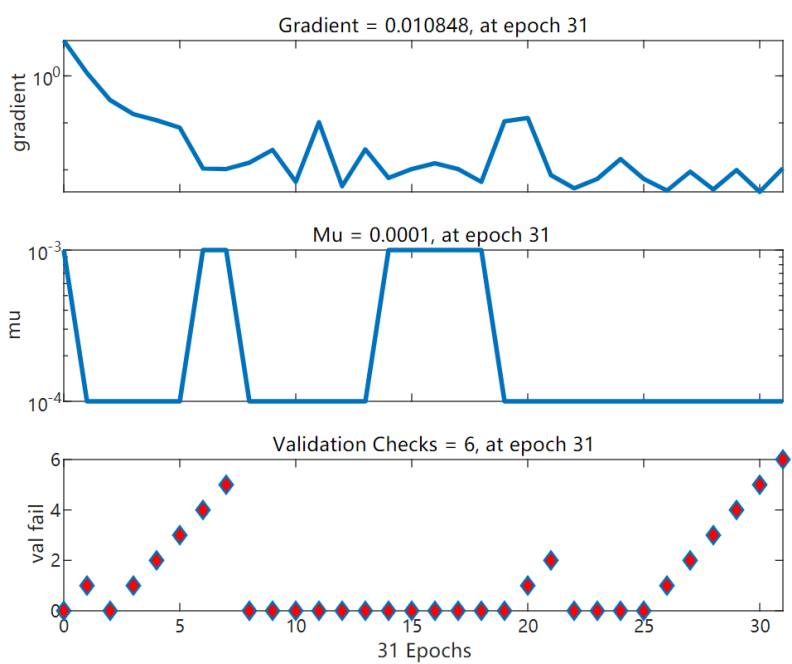

Figure 3. BP neural network training steps.

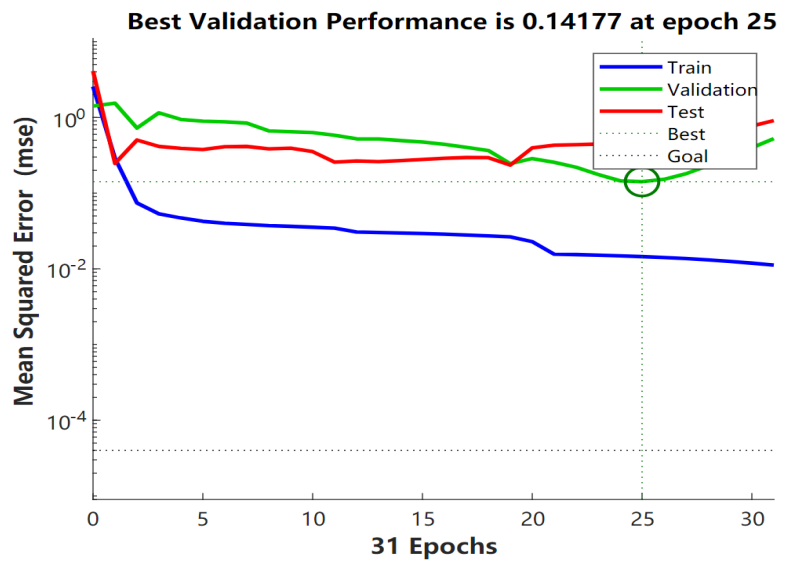

Figure 4. BP neural network training error.
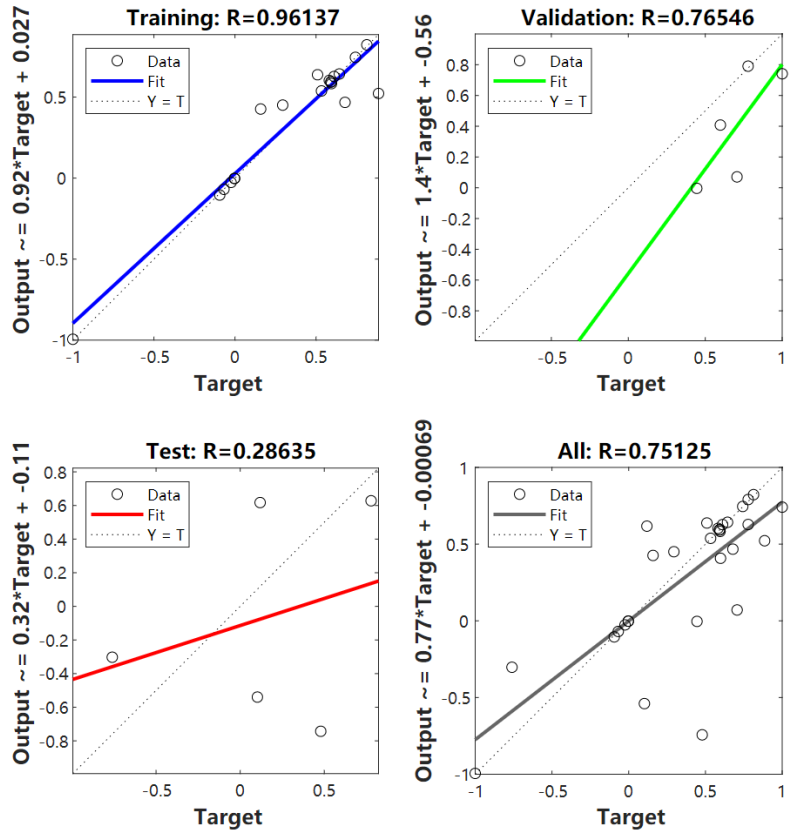

Figure 5. BP neural network goodness of fit.

In Figure 4, we can get the BP neural network training results, the training error is less than 0.1 , so the neural network training is good.

In Figure 5, the goodness of fit of the training reaches 0.9 , so our neural network results are reliable.

\section{Results and discussion}

The goodness of fit of the neural network can be obtained, and the training goodness of fit reaches 0.9 , so the result of our neural network is reliable. Visualize the predicted data on a map, as shown in the figure below:

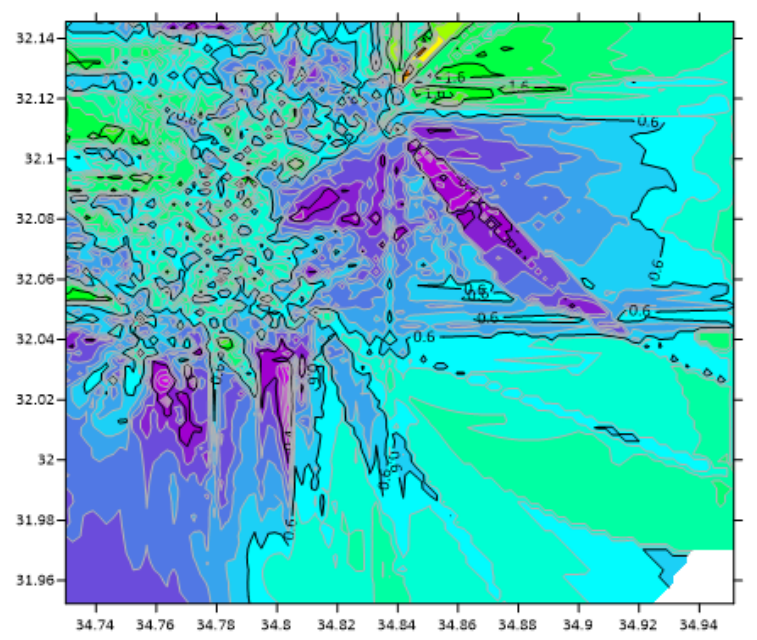

Figure 6. Plane visualization of parking demand forecast. 


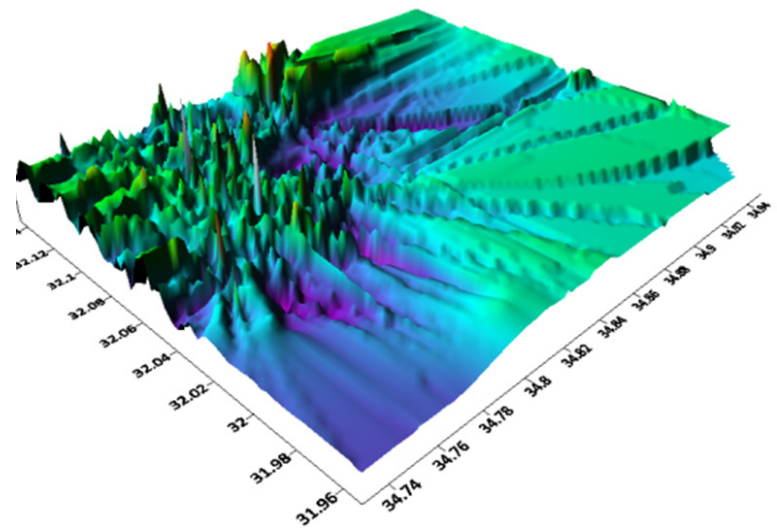

Figure 7. 3D visualization of parking demand forecast.

\begin{tabular}{l} 
Material Color \\
Upper \\
\hline
\end{tabular}

Figure 8. Change of color depth.

It can be seen from Figure 5 that the color area from left to right gradually turns from purple to red, which also means that the number of vehicles parked from left to right parking points is gradually increasing. The purple and blue areas indicate that the use of shared cars is not frequent, and the green Places indicate that the use of shared cars is average, and the yellow and red places indicate that the use of shared cars is frequent.

\section{6 conclusion}

This paper proposes a shared car scheduling method based on a neural network, which to some extent solves the problems of empty cars and empty seats, high platform scheduling costs, and imbalance between vehicle supply and demand. And the simulation results show that the user-participated car-sharing scheduling method proposed in this paper can reduce the total cost of the platform and increase the vehicle utilization rate, which provides new ideas for future research on carsharing service modes and scheduling methods.

\section{References}

1. Wei Z. Characteristic Analysis of Electric CarSharing System-Take EVCARD System as an Example. Communication \& Shipping, 2020.

2. Li L, Pantelidis T, Chow J, et al. A Real-Time Dispatching Strategy for Shared Automated Electric Vehicles with Performance Guarantees[J]. 2020.

3. Zhu H, Luo Y, Liu Q , et al. Multistep Flow Prediction on Car-Sharing Systems: A Multi-Graph Convolutional Neural Network with Attention Mechanism[J]. International Journal of Software Engineering and Knowledge Engineering, 2019, 29(11n12):1727-1740.

4. Guo G, Xu T. Vehicle Rebalancing With Charging Scheduling in One-Way Car-Sharing Systems[J].
IEEE Transactions on Intelligent Transportation Systems, 2020, PP(99):1-10.

5. Yang $\mathrm{S}, \mathrm{J} \mathrm{Wu}, \mathrm{Sun} \mathrm{H}$, et al. Double-balanced relocation optimization of one-way car-sharing system with real-time requests[J]. Transportation Research Part C Emerging Technologies, 2021, 125(9):103071. 